Article

\title{
CBL-interacting protein kinases (CIPKs) in Chickpea: Genome-wide identification, structure and expression analysis under abiotic stresses and development
}

\author{
Nikita Poddar ${ }^{1}$, Amarjeet Singh ${ }^{2, *}$ and Shailesh Kumar 1,* \\ 1 Bioinformatics Lab, National Institute of Plant Genome Research (NIPGR), Aruna Asaf Ali Marg, New \\ Delhi 110067, India. Tel: 91-11-26735217, Fax: 91-11-26741658, Email: shailesh@nipgr.ac.in \\ 2 National Institute of Plant Genome Research (NIPGR), Aruna Asaf Ali Marg, New Delhi 110067, India. \\ Phone: +91-11-26735224, Fax: +91-11-26741658, Email: amarjeet.singh@nipgr.ac.in \\ * Correspondence: SK (shailesh@nipgr.ac.in) and AS (amarjeet.singh@nipgr.ac.in)
}

\begin{abstract}
Calcineurin B-like proteins (CBL)-interacting protein kinases (CIPKs) by interacting with CBLs regulate developmental processes, hormone signalling transduction and mediate stress responses in plants. Although the genome of chickpea is available, information of CIPK gene family has been missing in chickpea. Here, a total of 22 CIPK encoding genes were identified in chickpea and characterized by in silico methods. We found a high structural conservation in chickpea CIPK family. Our analysis showed that chickpea CIPKs have evolved with dicots from common ancestors, and extensive gene duplication events have played an important role in evolution and expansion of CIPK family in chickpea. Most chickpea CIPK proteins localize in cytoplasm and nucleus. Promoter analysis revealed various cis-regulatory elements related to plant development, hormone signaling and abiotic stresses. Expression analysis indicated that CIPKs are significantly expressed in a spectrum of developmental stages, tissue/organs that hinted their important role in plant development. Several CIPK genes had specific and overlapping expressions in different abiotic stresses and seed development stages, suggesting the important role of CIPK family in abiotic stress signaling, and seed development in chickpea. Thus, this study provides the avenue for detailed functional characterization of CIPK family in chickpea and other legume crops.
\end{abstract}

Keywords: CIPK, Calcium, Chickpea, Structure, Phylogeny, Expression, Stress, Development.

\section{Introduction}

South Asia is the major producer of the world's second most important food legume chickpea. Importantly, India is the largest producer of chickpeas and is credited for about $70 \%$ of world's chickpeas production. India contributes an estimated production of 5.9 million tonnes (mt) annually [1]. Chickpea is an important dietary source for vegetarians due to vital nutritive constituents in its seeds, including $20-30 \%$ crude protein, $40 \%$ carbohydrate, and 3-6\% oil [2]. In addition, chickpea seeds are rich in minerals, such as calcium, magnesium, potassium, phosphorus, iron and zinc [3]. The chickpea production is severely affected by various stresses. Consequently, a huge gap is developed between its demand and supply. Abiotic stresses alone account for an estimated $40-60 \%$ global chickpea production losses annually. Drought causes major damage and accounts for about $50 \%$ of chickpea yield loss. Temperature fluctuations and soil salinity combined are responsible for about $25 \%$ of chickpea yield loss [4]. The chickpea yield loss due to drought, cold and salinity, respectively costed approximately 1.3 billion, 186 million and 354 million US dollars, which have economically dented several chickpea-producing countries [5]. These stresses have an adverse effect on flower set, pollen viability, pod set/abortion and retention. As all these crucial developmental stages essentially determine seed number, a negative impact on these stages significantly hampers chickpea yield. Due to 
continuous climate change, severe and frequent challenges of drought in arid and semiarid areas where chickpea is traditionally cultivated are predicted [6] and that can be detrimental for overall productivity of chickpea. Thus, identification and utilization of important stress related genes in biotechnological programmes to generate improved chickpea varieties is need of the hour.

Environmental cues, such as biotic and abiotic stresses are known to elicit the increase in cytosolic Ca2+ with specific spatio-temporal features. The spatio-temporal accumulation of $\mathrm{Ca} 2+$ generates specific "Ca2+ signature" in the form of spikes, waves and oscillations. The stimulus specific Ca2+ signature is decoded by $\mathrm{Ca} 2+$ sensors and downstream effectors towards a response [7]. Several Ca2+ sensors have been identified and characterized in plants, including calmodulin (CaM) and CaM-like proteins (CMLs) [8], $\mathrm{Ca} 2+$-dependent protein kinases (CDPKs) [9], and calcineurin B-like proteins (CBLs) [1]. Among these, CBLs are a unique group of $\mathrm{Ca} 2+$ sensors and to determine their functional identity, a family of plant specific serine/threonine kinases; CBL-interacting protein kinases (CIPKs) functions as important downstream signaling component [10]. Both CBLs and CIPKs are encoded as multi-gene families in higher plants, for example: $10 \mathrm{CBL}$ and 26 CIPKs members have been identified in Arabidopsis, and 10 CBL and 30 CIPKs in rice [11]. Large numbers of CBL and CIPK members in higher plants constitute a complex and sophisticated signaling network. For instance, In Arabidopsis, each CBL interacts with multiple CIPKs and vice-versa [12], consequently, some CBLs share a common CIPK partner and some CIPKs are regulated by a common CBL. Such specific and overlapping patterns of CBL-CIPK interactions may provide functional specificity and synergism to CBLCIPK signalling networks.

Structurally, CBLs are typical Ca2+ sensor proteins with four EF-hand domains which are responsible for $\mathrm{Ca} 2+$ binding. On the other hand, CIPKs harbours several functionally distinct domains. All CIPKs consist of a conserved catalytic kinase domain at the $\mathrm{N}$-terminal and a regulatory domain at the C-terminal [10,12]. A typical of a functional kinase protein, CIPK kinase domain contains an ATP binding site and an activation loop. The regulatory domain contains FISL/NAF and PPI motifs which are responsible for the interaction with CBL and type $2 \mathrm{C}$ protein phosphatases, respectively $[13,14]$. The function of CBL-CIPK pathways could be regulated by pattern of gene expression, Ca2+ binding affinity, protein stability and protein-protein interactions [7]. CBL-CIPK networks have been implicated in diverse functions that regulate plant response to biotic stress [15-17], abiotic stress [1, 18, 19], nutrient deficiency [1, 20, 21] metal toxicity [22, 23] and plant development [24-27]. Majority of the knowledge about CBL-CIPK signaling has developed from the research with model plant Arabidopsis thaliana. Information about CBLCIPK networks and their role is scarce in important legume crop chickpea. Though, CBL family has been identified in chickpea [28], identification and characterization of CIPK family is missing. A comprehensive gene expression profiling of the CIPK family will help in understanding CBL-CIPK functions in chickpea. Information obtained from expression analysis will encourage the utilization of crucial genes for genetically engineering the chickpea plant towards better stress tolerance and development.

With this rationale, we have identified the CIPK gene family in chickpea. Phylogenetic analysis and chromosomal localization have provided insight into the evolution and expansion of chickpea CIPK family. Analysis of gene and domain structure ensured the authenticity and integrity of identified genes. Homology modelling helped to understand the three-dimensional structure of chickpea CIPKs. In-silico analysis revealed various stress, hormone and development related cis-regulatory elements in CIPK promoters. Expression profiling using various datasets in public repositories suggested involvement of the CIPK family in biotic and abiotic stress signaling, and seed development in chickpea. 


\section{Materials and Methods}

\subsection{Identification of CIPKs in the chickpea genome}

The chickpea genome submitted by Varshney et al. (2013) was downloaded from the NCBI and explored to identify CIPK encoding genes. Rice and Arabidopsis thaliana CIPK proteins were retrieved from Uniprot (Swiss-Prot), and homology search was performed using BLAST tool (E-value =10-6) against the chickpea proteome. Significant hits were selected on the basis of $>=50 \%$ identity, and $>=100$ amino acid length alignment. Further, the HMM sequence of CIPK-NAF domain was extracted from Pfam (http://pfam.xfam.org/) database, and BLAST search was done $(E-v a l u e=10)$ against the chickpea proteome. Furthermore, both the sets of putative candidates were mixed, and redundant sequences were removed using CD-HIT [29]. The domain analysis was performed by using a standalone version of the InterproScan [30]. The gene attributes such as gene ID, protein ID, CDS, size of amino acid and chromosomal coordinates were extracted from NCBI web server.

\subsection{Phylogenetic analysis}

To examine the evolutionary relationship between CIPKs in chickpea and other species, Multiple Sequence Alignment (MSA) was performed with the amino acid sequences of CIPKs from four different plant species e.g., Arabidopsis thaliana, Oryza sativa, Glycine max, and Cicer arietinum) using ClustalW [31] at default settings in MEGA X version 10.1.8 [32]. The neighbour-joining method was used to construct the phylogenetic tree and bootstrap values were calculated in 1000 replicates to determine the phylogenetic relationship among the CIPKs. iTOL [33] webserver was used to mark the different clades of CIPKs with different colours and shapes for better visualization.

\subsection{Gene structures, motif organization and domain prediction}

Gene Structure Display Server (GSDS) program (http://gsds.gao-lab.org/) was used to compare the CDS sequences with their corresponding genomic DNA sequences in order to investigate the coding sequences and intron structure. Motif organization of CIPK proteins was examined via the Multiple Expression motifs for Motif Elicitation (MEME) tool [34] with default parameters; site distribution - zero or one occurrence per sequence; motif discovery mode - classic; motif length 6-50; and the top ten most enriched motifs were selected based on lowest E-values. The identification of domains was performed by a standalone package of InterProScan [30]. The coordinates of the essential domains and active sites were extracted and used as input in Illustrator for Biological Sequences [35] for the visualization.

\subsection{Gene nomenclature, chromosomal location and gene duplication}

The names of CIPK genes in chickpea were assigned according to their closest orthologous relationship with Arabidopsis CIPK genes in the phylogenetic tree. The information of the chromosome coordinates was obtained from NCBI. Their localization was displayed in different chromosomes using TBtools [36]. To search for all duplicated gene pairs within the chickpea genome, the protein sequence of chickpea was used to run the all-versus-all local BLASTP with parameters of E-value 1e-5, max target sequences 5, and m6 format output. MCScanX software package [37] was used to analyse the segmentally duplicated regions of CIPK genes of chickpea. The genes and the intra-species collinear gene pairs were mapped to the eight chromosomes of chickpea using the family_circle_plotter.java script. The protein sequences of each duplicate gene pair were aligned by CLUSTALW. The alignment file in FASTA format and the CDS sequences of the 
corresponding genes were used to calculate the non-synonymous $(\mathrm{Ka})$ and synonymous (Ks) substitution values by the PAL2NAL server [38].

\subsection{In silico promoter analysis}

For the identification of cis-regulatory elements in the promoters of chickpea CIPKs, $2000 \mathrm{bp}$ upstream sequences of the coding region of genes were extracted from NCBI and used as input in the PlantCARE (http://bioinformatics.psb.ugent.be/webtools/plantcare/html/) tool.

\subsection{Subcellular localization and physicochemical properties of CaCIPK proteins}

The full-length protein sequences of all the CIPKs of chickpea were used as input to predict their subcellular localization using the CELLO program [39]. The locations were displayed in different parts of the cell by Biorender software (https://biorender.com/). The online tool Compute pI/MW of Expasy [40] was used to calculate the molecular weight (MW) and isoelectric point (pI) of CaCIPKs.

\subsection{Protein-Protein Interaction Network Construction for CaCBLs-CaCIPKs}

To elucidate the interaction network between CIPK and CBL proteins in chickpea, the amino acid sequences of 22 CIPKs, and 9 CBLs from the study of Meena et al., 2015 were used as input in STRING (http://string-db.org/). At STRING, the interaction network can be constructed using low confidence value of 0.15 , medium confidence of 0.4 , high confidence of 0.7 and highest confidence of 0.9 . Experimental data of interacting CBL and CIPK proteins in Arabidopsis were constructed using the confidence value $>0.4$. Homologous proteins of the determined interactive Arabidopsis proteins in chickpea were identified by reciprocal best BLASTP analysis.

\subsection{Protein tertiary structure prediction}

The tertiary structures of the 22 CaCIPK proteins were predicted with the Phyre2 web portal (http://www.sbg.bio.ic.ac.uk/phyre2). Phyre2 uses advanced remote homology detection methods to build 3D models for protein sequences [41]. All the proteins were modelled with $100 \%$ confidence by the single highest scoring template model.

\subsection{Expression analysis using $R N A$-seq data}

To generate the genome-wide expression profiles of CaCIPK genes in different tissues and developmental stages, RNA-Seq data was extracted from NCBI Sequence Read Archive; SRA number SRP121085. RNA-seq data for different seed stages in two distinct desi chickpea varieties (JGK3 and Himchana 1) was extracted from SRA number SRP072563 and SRP072564. Data for three abiotic stresses (desiccation, cold and salinity stress) in root, and shoot tissue of ICC4958 chickpea variety was extracted from SRA number SRP034839.

The raw reads downloaded from SRA at NCBI were processed using FASTP [42] to remove the adapter, poly-N, short and low-quality reads. The reference genome of chickpea was downloaded from the NCBI genome web server. The HISAT2 [43] tool was used for building the index of the reference genome, and for mapping of filtered reads onto the genome. The alignments were assembled into potential transcripts using StringTie [44] and the transcript abundance was calculated as fragments per kilobase of transcript per million reads (FPKM) values. For differential expression analysis, three biological replicates of each treatment and control were analysed and fold change expression was 
calculated by the ratio of average FPKM of test samples and average FPKM of control samples. The 'pheatmap' package of $R$ was used to generate the heatmaps of the expression data using the logarithm of normalized expression values for the tissue study and the logarithm of fold change for the remaining studies.

\section{Results and Discussions}

\subsection{Identification and sequence analysis of CaCIPK genes}

A total of 39 putative CIPK sequences were obtained from homology search with Arabidopsis and rice CIPKs. Further HMM profile search against chickpea proteome revealed 38 putative CIPK sequences. After combining both sets, followed by manual curation a total of 26 unique CIPK sequences were obtained. Domain analysis revealed that the necessary domains (e.g. PPI and NAF domain) were absent in four sequences, therefore, they were removed from the list. Finally, a total of 22 non-redundant CIPK encoding genes were found in the chickpea genome. Previously, 26 CIPK members have been reported in Arabidopsis [45] and 33 in rice [46]. The number of CIPKs in chickpea is comparable with wheat (20 members), tomato (22 members) and canola (23 members) [47-49].

The length of 22 CaCIPK proteins varied from 418aa (CaCIPK16) to 503aa (CaCIPK12) with average molecular weight of $51.16 \mathrm{kDa}$. Most of the CaCIPK proteins (except CaCIPK3 and CaCIPK11) were found to have an isoelectric point (pI) greater than 7 (Table 1).

Table 1: Various features of the chickpea CIPK family.

\begin{tabular}{|c|c|c|c|c|c|c|c|c|c|c|}
\hline \multicolumn{5}{|c|}{ Transcript } & \multicolumn{6}{|c|}{ Protein } \\
\hline $\begin{array}{l}\text { Gene } \\
\text { Name }\end{array}$ & NCBI ID & $\begin{array}{l}\text { Chrom } \\
\text { osome }\end{array}$ & Start & Identifier & $\begin{array}{l}\text { Intron } \\
\text { count }\end{array}$ & $\begin{array}{c}\text { CDS } \\
\text { length }\end{array}$ & Identifier & $\begin{array}{c}\text { Length } \\
\text { (aa) }\end{array}$ & $\begin{array}{l}\text { Isoelectric } \\
\text { point (pI) }\end{array}$ & $\begin{array}{c}\text { Protein wt. } \\
\text { (kDa) }\end{array}$ \\
\hline CIPK1 & LOC101499928 & $\mathrm{Ca} 1$ & 25702213 & 25709285 XM_004488130.3 & 11 & 1347 & XP_004488187.1 & 448 & 8.02 & 50.34 \\
\hline CIPK2 & LOC101488582 & $\mathrm{Ca} 2$ & 27180279 & 27182763 XM_004490648.3 & 0 & 1368 & XP_004490705.1 & 455 & 9 & 51.9 \\
\hline CIPK3 & LOC101491417 & $\mathrm{Ca} 2$ & 33011673 & 33018054 XM_004491146.3 & 13 & 1326 & XP_004491203.1 & 441 & 6.59 & 50.44 \\
\hline CIPK4 & LOC101504371 & $\mathrm{Ca} 4$ & 32563304 & 32564889 XM_004497948.3 & 0 & 1305 & XP_004498005.1 & 434 & 9.12 & 49.29 \\
\hline CIPK5 & LOC101513526 & $\mathrm{Ca} 6$ & 53224442 & 53226557 XM_012717669.2 & 0 & 1353 & XP_012573123.1 & 450 & 8.26 & 51.83 \\
\hline CIPK6 & LOC101511702 & $\mathrm{Ca} 7$ & 1293373 & 1294939 NM_001309656.1 & 0 & 1335 & NP_001296585.1 & 444 & 9.11 & 50.34 \\
\hline CIPK 7 & LOC101496895 & $\mathrm{Ca} 1$ & 8839486 & 8841422 XM_004486556.3 & 0 & 1347 & XP_004486613.1 & 448 & 9.14 & 50.77 \\
\hline CIPK8 & LOC101511605 & $\mathrm{Ca} 2$ & 23829223 & 23836498 XM_027331998.1 & 14 & 1425 & XP_027187799.1 & 474 & 8.03 & 53.78 \\
\hline CIPK9 & LOC101493574 & $\mathrm{Ca} 1$ & 36737555 & 36744930 XM_027334254.1 & 14 & 1398 & XP_027190055.1 & 465 & 8.93 & 52.14 \\
\hline CIPK10 & LOC101498077 & $\mathrm{Ca} 5$ & 26642943 & 26645602 XM_004500288.3 & 0 & 1392 & XP_004500345.1 & 463 & 8.76 & 52.43 \\
\hline CIPK11 & LOC101499591 & $\mathrm{Ca} 5$ & 26665837 & 26667956 XM_012715825.2 & 0 & 1320 & XP_012571279.1 & 439 & 6.49 & 48.88 \\
\hline CIPK12 & LOC101506657 & $\mathrm{Ca} 1$ & 3314537 & 3316964 XM_012712534.2 & 0 & 1512 & XP_012567988.1 & 503 & 7.14 & 56.66 \\
\hline CIPK13 & LOC 101489428 & $\mathrm{Ca} 5$ & 28720785 & 28723545 XM_004500487.3 & 0 & 1380 & XP_004500544.1 & 459 & 9.04 & 51.78 \\
\hline CIPK14 & LOC 101488926 & $\mathrm{Ca} 2$ & 27205251 & 27207496 XM_004490649.3 & 1 & 1308 & XP_004490706.1 & 435 & 8.67 & 48.82 \\
\hline CIPK15 & LOC101507945 & $\mathrm{Ca} 6$ & 37592424 & 37596217 XM_004506380.3 & 0 & 1395 & XP_004506437.1 & 464 & 8.82 & 52.36 \\
\hline CIPK16 & LOC 101510050 & $\mathrm{Ca} 4$ & 44315144 & 44317032 XM_004498761.3 & 0 & 1257 & XP_004498818.1 & 418 & 8.94 & 47.54 \\
\hline CIPK17 & LOC101510187 & $\mathrm{Ca} 7$ & 3876530 & 3882455 XM_012717926.2 & 11 & 1344 & XP_012573380.1 & 446 & 8.43 & 50.8 \\
\hline CIPK18 & LOC101492060 & $\mathrm{Ca} 3$ & 21042046 & 21046759 XM_004492487.3 & 0 & 1392 & XP_004492544.1 & 463 & 8.41 & 52.63 \\
\hline CIPK19 & LOC101507330 & $\mathrm{Ca} 7$ & 6850555 & 6859747 XM_012718074.2 & 13 & 1341 & XP_012573528.1 & 446 & 9.2 & 50.84 \\
\hline
\end{tabular}




\begin{tabular}{llllllllllll}
\hline CIPK20 & LOC101506991 & Ca1 & 3307254 & 3309153 & XM_012712535.2 & 0 & 1362 & XP_012567989.1 & 453 & 8.96 & 52.14 \\
\hline CIPK21 & LOC101512343 & Ca7 & 2026868 & 2030503 & XM_012717974.2 & 13 & 1359 & XP_012573428.1 & 452 & 8.17 & 51.07 \\
\hline CIPK22 & LOC101493164 & Ca3 & 21099833 & 21101565 & XM_004492489.3 & 0 & 1296 & XP_004492546.1 & 431 & 8.89 & 48.89 \\
\hline
\end{tabular}

This indicates that most CaCIPK proteins function in similar microenvironments. Similar structural features and functional $\mathrm{pI}$ had been previously reported in CIPKs of other plants such as P.mume [50], C.annum [51] and B.napus [49]. To gain insights into the homology of the CaCIPK proteins, the sequence identity and similarity was calculated by the SIAS tool (http://imed.med.ucm.es/Tools/sias.html). The analysis showed that different CaCIPKs have 46.13 to $81.87 \%$ sequence similarity among themselves. Four protein pairs; CaCIPK15/18, CaCIPK5/7, CaCIPK1/17 and CaCIPK2/10) showed a high degree of identity i.e., $76.67 \%, 76.39 \%, 76.01 \%$ and $73.84 \%$, respectively (Figure S1). Even the most divergent protein pair CaCIPK8 and CaCIPK22 shared 35.49\% identity (49.18\% similarity). These findings suggest that plant CIPKs are highly conserved in terms of sequence and structure, which hints towards their similar mode of action.

\subsection{Gene and domain structure}

The evolution of gene families is often reflected by their gene structure [52, 53]. A large variation in the number of introns was found in CIPK genes in chickpeas with number of introns ranging from 0 to 14 (Figure 1A). Out of $22 \mathrm{CaCIPK}$ genes, only seven had more than two introns. Thus, CaCIPKs could be classified into two groups: i) intron-poor subgroup with zero (CaCIPK2, -4, -5, -6, -7, -10, -11, -12, -13, -15, -16, -18, -20, -22) or one (CaCIPK14) intron, and ii) intron-rich subgroup with greater than 10 introns (CaCIPK1, $3,-8,-9,-17,-19,-21)$. Division of CIPKs into two subgroups was also supported by the division of clade in the phylogenetic tree. Similar pattern of intron-rich and intron-poor CIPK genes has been reported in different plant species, including Arabidopsis [54], rice [55], soybean [56], wild sugarcane [57] and wheat [47]. These findings suggest that CIPK gene family is structurally conserved across plant kingdom.

(A)

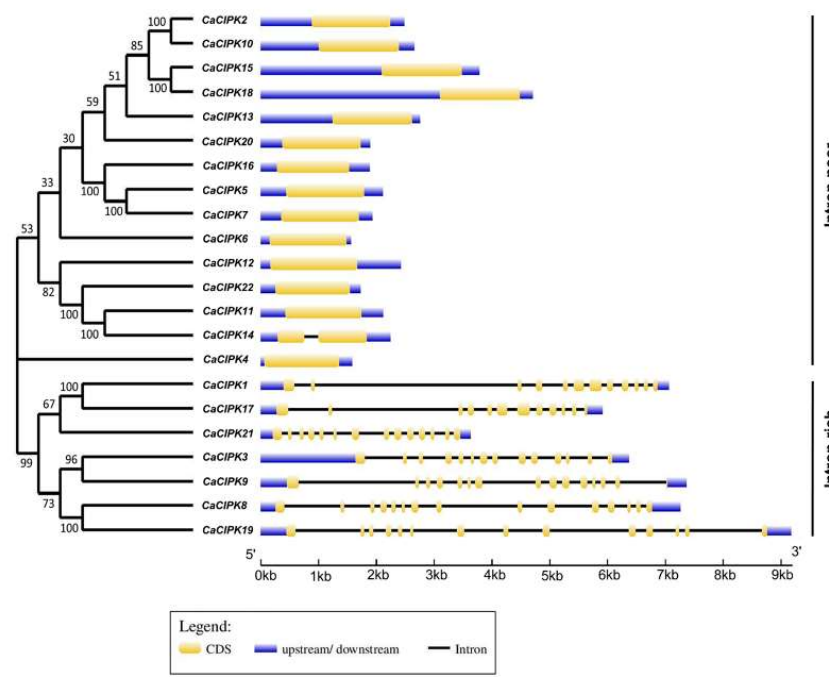

(B)

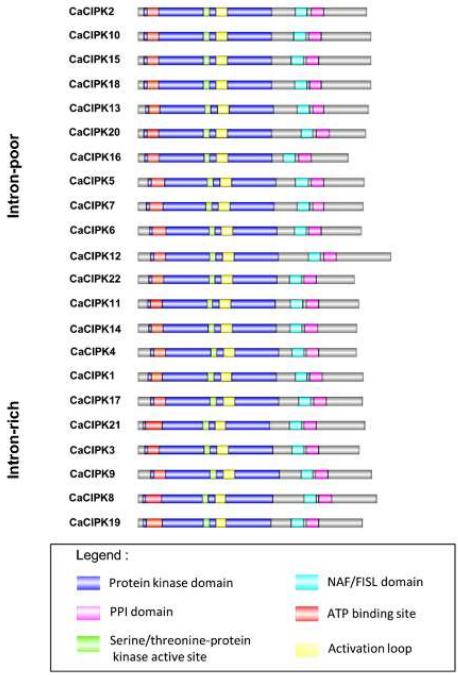

Figure 1. Structural features of chickpea CIPK family. (A) Exon-intron organization is shown for $22 \mathrm{CaCIPK}$ genes. The values in the phylogenetic tree (left side) represent 
bootstrap values. (B) Protein structure of chickpea CIPK family is showing conserved protein kinase, NAF and PPI domain along with some important sites present at the N-terminal e.g. ATP binding site, Serine/threonine-protein kinase active site and activation loop.

The domain structure analysis revealed that all the CaCIPK proteins possess three essential domains, at N-terminal a kinase domain, and at C-terminal regulatory NAF and protein phosphatase interaction (PPI) domain (Figure 1(B)). Kinase domains contain an ATP binding site and an activation loop. During CIPK activity, the stabilization of substrates at the active site is regulated by the phosphorylation of activation loop [58]. The activation loop has been found between the conserved 'DFG' and 'APE' amino acid residues. However, in our analysis, few variations were observed in the conserved short motifs. Alignment of $22 \mathrm{CaCIPK}$ proteins showed that the glycine residue of 'DFG' was changed to asparagine (DFN) in CaCIPK1, whereas the alanine of 'APE' was modified to serine (SPE) in CaCIPK6 and CaCIPK18 (Figure S2). Importantly, sites important for phosphoregulation of activation loop i.e., serine, threonine, and tyrosine [59] were found to be conserved in all CaCIPK proteins except CaCIPK6 where serine was modified to cysteine. Further investigations are required to assess an effect of these changes on the function of the activation loop. In CIPKs, NAF motif mediates the interaction between CIPK and CBL, and FISL motif keeps the kinase inactive under normal conditions, hence act as autoinhibitory domain $[13,14]$. PPI domain is required for interaction of CIPKs with protein phosphatase 2C (PP2C) [60]. In CaCIPK proteins, a total of 10 conserved motifs were identified by using the MEME tool. Out of those, motif 7 was annotated as NAF domain due to the presence of conserved asparagine-alanine-phenylalanine residues [13], whereas motif 8 which is located just after the motif 7 was designated as the PPI domain as it contains important arginine and phenylalanine residues. All motifs except motif 6 and 10 were present in 22 CaCIPK proteins (Figure S3). Motif 6 was absent in CaCIPK4, whereas motif 10 was absent in the subgroup of intron-rich CaCIPKs. This may explain the presence of CaCIPK4 in a separate phylogenetic clade. The similar motif composition of proteins of the same clade was also reported in tomato and saccharum $[48,57]$. Furthermore, the sequential arrangement and the size of motifs in all the CaCIPK proteins were similar, which hints towards structural conservation and a close phylogenetic relationship, as previously reported in Prunus mume [50]. The sequence logo of different motifs is depicted in Table S1.

\subsection{Phylogenetic analysis of CaCIPK family}

A total of 133 CIPK protein sequences from four species: Arabidopsis thaliana (26), Oryza sativa (33), Glycine max (52), and Cicer arietinum (22) were used to construct the phylogenetic tree to explore the evolutionary relationship among the CIPKs. Based on high bootstrap values, the tree was divided into two major groups: group I and II. Two groups were further sub-divided IA, IB, and IIA, IIB (Figure 2). 


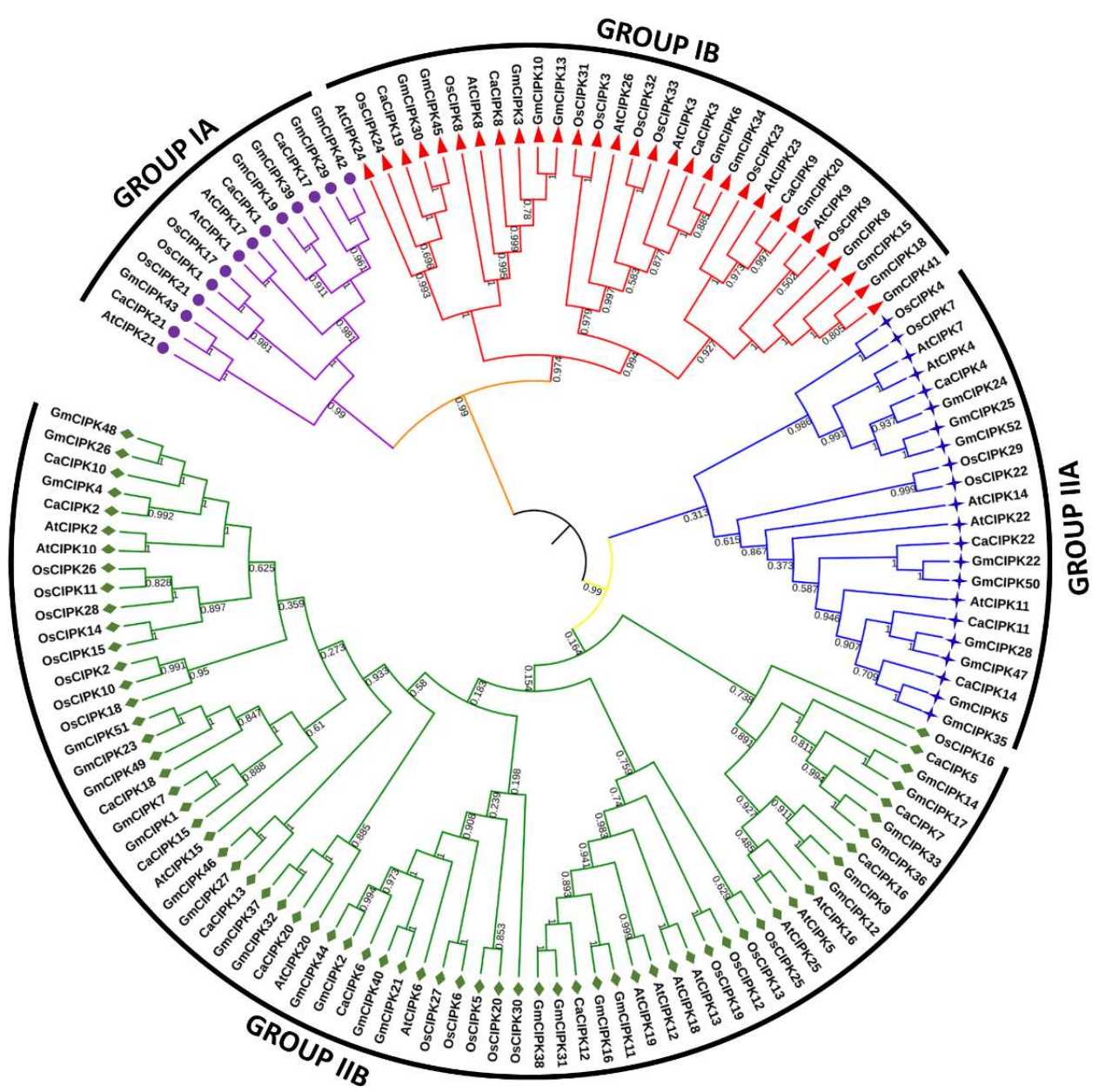

Figure 2. Phylogenetic relationship among CIPKs from different plants. The evolutionary relationship from Arabidopsis thaliana (At), Oryza sativa $(\mathrm{Os})$, Glycine max $(\mathrm{Gm})$ and Cicer arietinum (Ca) constructed using Neighbor-Joining method is shown via phylogenetic tree. Labels above the nodes represent bootstrap values calculated from 1000 replicates. The Group IA, IB, IIA and IIB are indicated by violet, red, blue and green branch lines respectively.

Interestingly, all intron-rich CaCIPKs were clustered in group I, and intron-poor $\mathrm{CaCIPKs}$ were clustered in group II. This indicates evolutionary conservation among intron-rich and intron-poor CIPKs in chickpea, and thus CIPKs may have evolved as two groups in chickpea. Similar phylogenetic pattern has been observed for CIPKs in other plants, such as Zea mays [61] and Prunus mume [50]. Remarkably, CaCIPKs were found to be close to CIPKs from Arabidopsis and soybean, but distantly placed from rice CIPKs. This suggests that CIPKs might have evolved separately in dicots and monocots. Group IIB contained most members of CaCIPKs. Group IA includes CaCIPK1, -17, -21, group IB includes CaCIPK3, $-8,-9$ and -19, Group IIA includes CaCIPK4, -11, -14 and -22 and Group IIB includes CaCIPK2, -5, -6, -7, -10, -12, -13, -15, -16, -18 and -20.

\subsection{Chromosomal location and gene duplication of CaCIPK genes}

All the CaCIPK genes were mapped onto the seven out of eight chromosomes of chickpea. None of them was localized on chromosome 8 . All the genes were unevenly 
distributed on the seven chromosomes (Figure 3). Chromosome 1 contains the maximum number of genes viz. CIPK1, -7, -9, -12, -20. Chromosome 2 and 7 harbours four genes each i.e., CIPK2, -3, -8, -14 and CIPK6, -17, -19, -21 respectively. Chromosome 5 contained three genes (CIPK10, -11, -13) that were located very close to each other. The other chromosomes comprised only two CIPKs each. The uneven distribution pattern of CIPKs in chickpea is consistent with previous reports of CIPKs in tomato, pepper, sorghum, rice, apple and woody plant $[48,50,51,55,62,63]$.
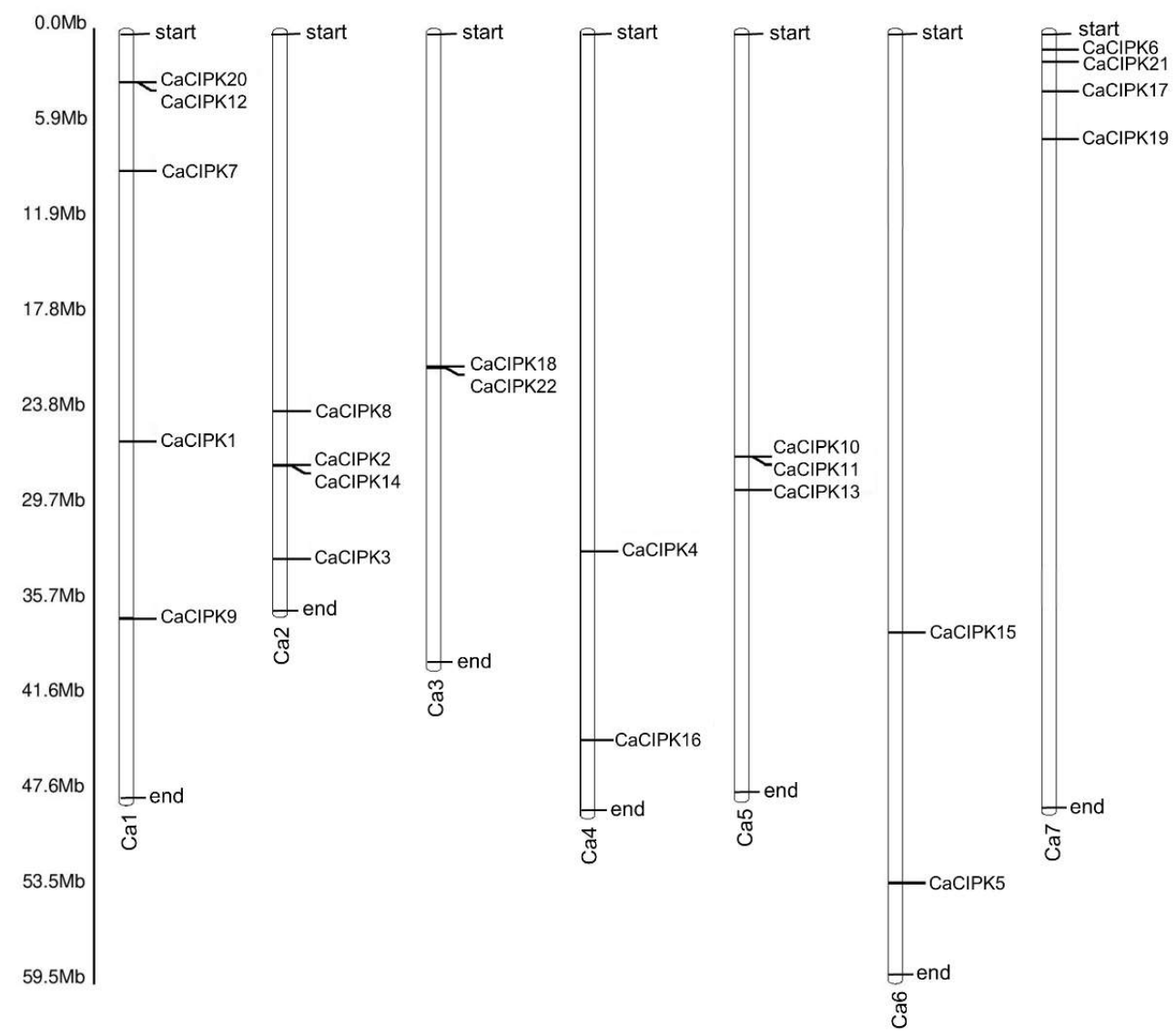

Figure 3. Chromosomal locations of chickpea CIPK genes. The 22 CIPK genes of chickpea were mapped to different chromosomes using TBtools.

CaCIPK family has undergone gene duplication as nine gene pairs showed segmental duplications which include CaCIPK1/17, CaCIPK2/10, CaCIPK2/18, CaCIPK5/7, CaCIPK10/18, CaCIPK11/14, CaCIPK11/22, CaCIPK14/22 and CaCIPK15/18 (Figure S4). In addition, four gene pairs (CaCIPK12/20, CaCIPK2/14, CaCIPK18/22, CaCIPK10/11) showed tandem duplications. Similarly, CIPK gene family has been found to exhibit significant gene duplication in plant species like Arabidopsis, rice and Vitis vinifera [64-66]. This suggests that gene duplication has been the major driving force behind evolution and expansion of CIPK gene family in chickpea and other plants. A ratio of Ka (non-synonymous) $/ \mathrm{Ks}$ (synonymous substitution) $=1$ signifies neutral selection (drift), $\mathrm{Ka} / \mathrm{Ks}<1$ indicates purifying selection and $\mathrm{Ka} / \mathrm{Ks}>1$ implies positive selection (adaptive evolution) [67]. In our study, the ratio of $\mathrm{Ka}$ to $\mathrm{Ks}$ for CaCIPKs was calculated which ranged from 0.0068 to 0.1675 . Thus, $\mathrm{Ka} / \mathrm{Ks}$ was found to be less than 1 for all segmentally duplicated gene pairs which suggests that all duplicated genes of CaCIPK family had undergone purifying selection on the whole genome duplication (WGD) (Table S2). 


\subsection{Cis-regulatory elements in CaCIPK promoters}

Various cis-regulatory elements were found to be unevenly distributed on CaCIPK promoters (Figure 4).

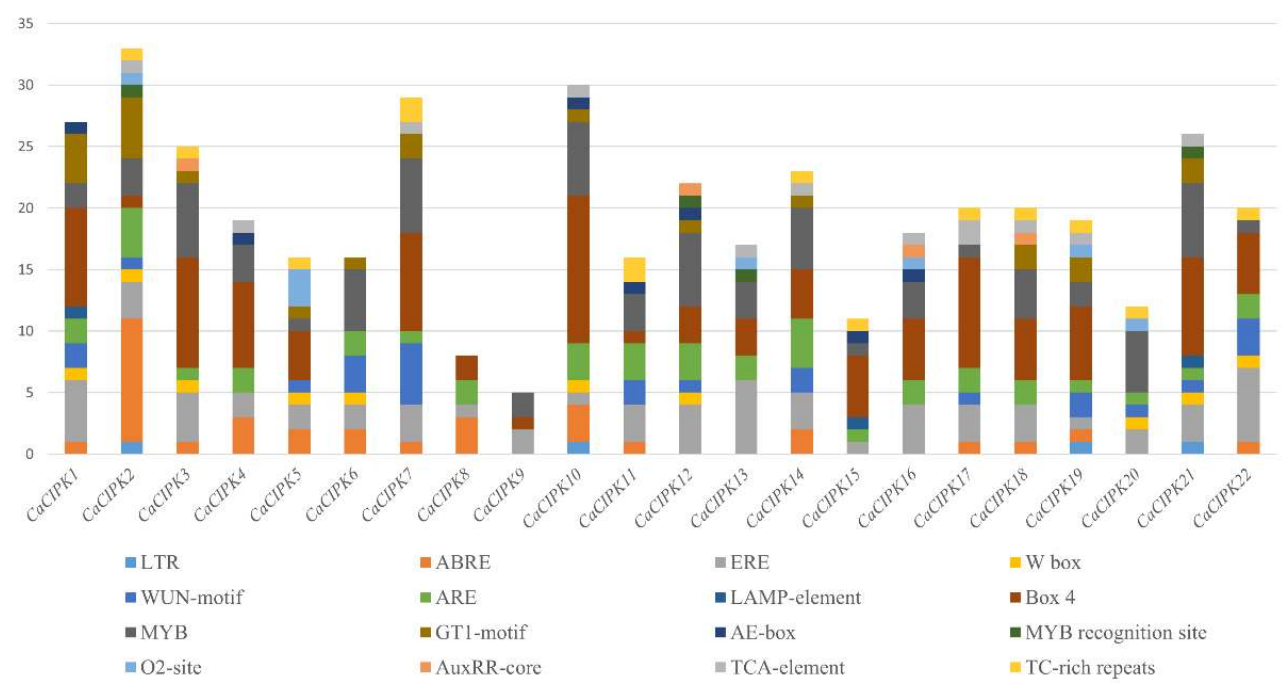

Figure 4. Different cis-regulatory elements in the $2 \mathrm{~kb}$ upstream region of $22 \mathrm{CaCIPK}$ genes are illustrated by different colours in the bar chart. The $X$-axis represents the name of genes and $\mathrm{Y}$-axis represents the number of cis-elements in each promoter. The names of cis-regulatory elements are mentioned below the graph.

These cis-elements have been found to be associated with different stress responses, including ARE (anaerobic induction), MYB (drought stress), LTR (low-temperature responsiveness), WUN-motif (wound-responsiveness), MYB recognition site (drought stress), responsive to phytohormones, such as ERE (ethylene responsive element), ABRE (abscisic acid responsiveness), W-box (WRKY transcription factor binding site), TCA-element (salicylic acid responsiveness), AuxRR-core (auxin responsive element), and, O2-site (cellular development), TC-rich repeats (defence and stress responsiveness) and Box4, GT1-motif, LAMP-element (light responsiveness) [68-74] . Apart from these, an oxidative stress responsive element ERE [75] was found to be present in all CaCIPKs. W box has a role in both biotic and abiotic stress [76], and was present in CaCIPK1, -2, -3, -5, -6, -10, $12,-20,-21,-22$. MYB was present in all the CaCIPK genes except CaCIPK8. O2 site involved in zein metabolism and circadian motif [75], was present in only six CaCIPK genes viz. CaCIPK2, -5, -13, -16, -19, -20. Another well characterized cis-element, ABRE involved in abiotic stress and $\mathrm{ABA}$ responsiveness $[77,78]$ was found in the promoter of $15 \mathrm{CaCIPK}$ genes, including CaCIPK1, -2, -3, -4, -5, -6, -7, -8, -10, -11, -14, -17, -18, -19, -22. Besides, LTR-motif was one of the least common elements and only four CaCIPK genes (CaCIPK2, $-10,-19,-21)$ contained this motif (Table S3). Overall, presence of different cis-regulatory elements in CaCIPK promoters indicate their role in abiotic and biotic stress responses, phytohormone signaling and plant development.

\subsection{Subcellular localization and structure prediction}

In our study, the majority of CaCIPK proteins were found to localize in the cytoplasm, and only six CaCIPK proteins were localized in the nucleus (Figure 5). Among 22 CIPKs, five proteins namely CaCIPK2, $-6,-10,-13,-19$ were found to be localized both in the nucleus and cytosol. 


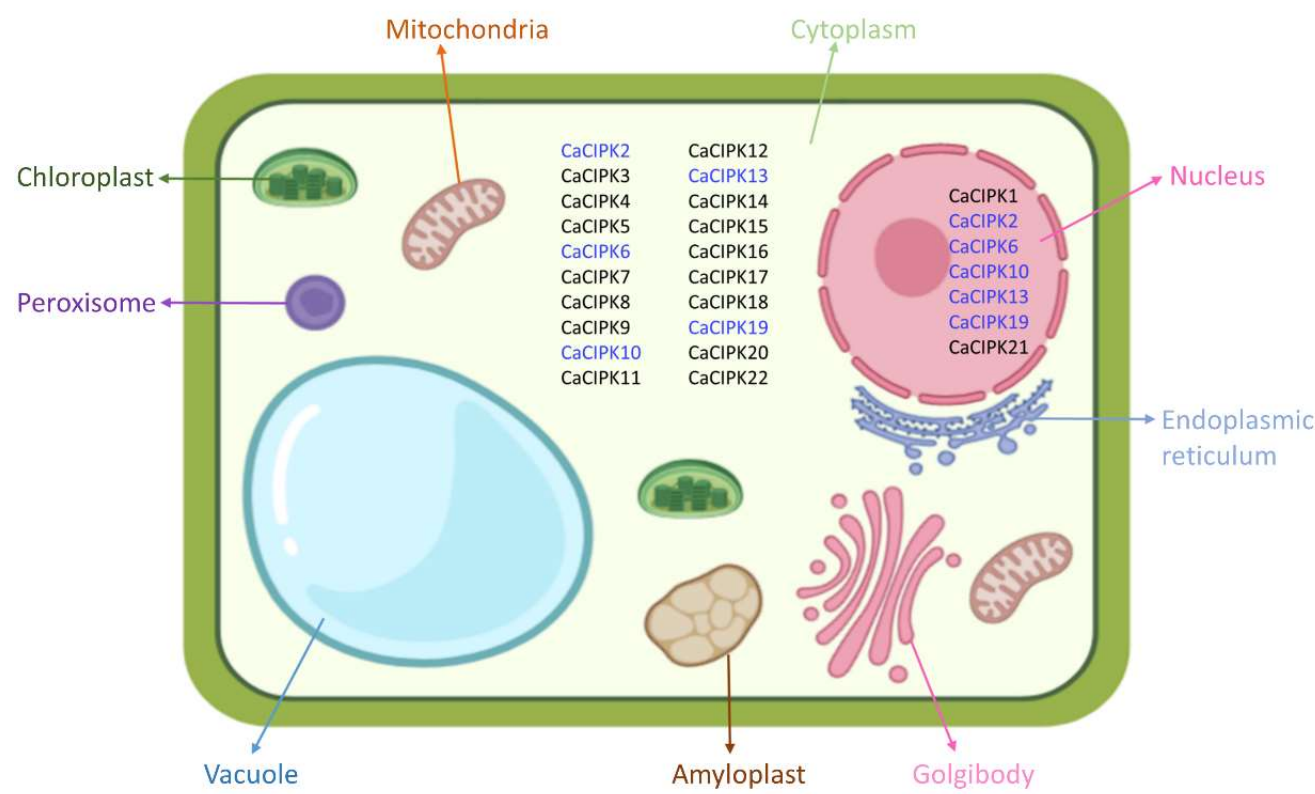

Figure 5. Subcellular localization of chickpea CIPK proteins predicted using the CELLO program. Genes located in both cytoplasm and nuclei are indicated by blue font.

Till now, the subcellular localization of few CIPKs in Arabidopsis has been reported and they have been localized in cytoplasm and nucleus [79, 80]. However, subcellular localization of the majority of CIPKs in other plants such as rice, maize and soybean has not been determined yet. In general, CBLs recruit CIPKs to plasma membranes or tonoplasts to perform their different functions. For example, AtCBL10 recruits AtCIPK24 at the tonoplast to regulate the vacuolar homeostasis of Na+ [81]. AtCBL1 and AtCBL9 recruit AtCIPK1 to the plasma membrane whereas, AtCBL2 recruit to the tonoplast $[82,83]$ Interestingly, AtCBL1 and AtCBL9 also recruit AtCIPK23 at the plasma membrane [84]. Therefore, future investigation of $\mathrm{CaCBL}-\mathrm{CaCIPK}$ interactions under different conditions will reveal their exact subcellular localization.

The three- dimensional structures of 22 CaCIPK proteins were modelled with $100 \%$ confidence by the single highest scoring template (Figure 6). 

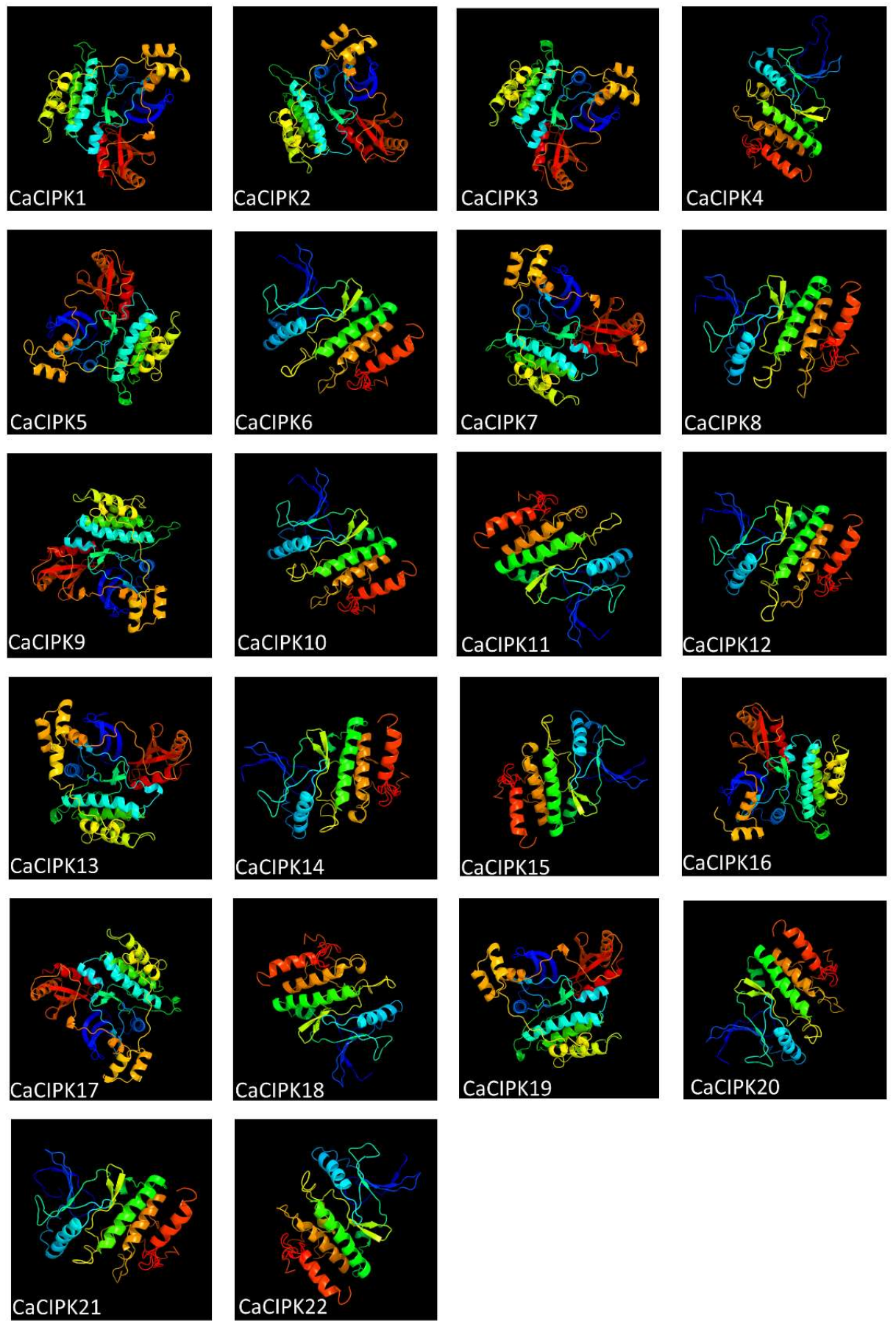

Figure 6. Predicted tertiary structures of chickpea CIPK proteins by PHYRE2.

Majority part of the models were built based on two templates - c6c9dB (Serine/threonine-protein kinase MARK1) and c5ebzF (inhibitor of nuclear factor kappa-b kinase subunit alpha) on the basis of raw alignment score which takes into account sequence and secondary structure similarity, inserts and deletes. CaCIPK16 showed the maximum coverage (95\%) whereas CaCIPK12 showed the least coverage (50\%). The identity of the template model c4czuC which belonged to CIPK23 with other CIPKs varied from $52 \%$ to $85 \%$ 
(Table S4). All the CaCIPK proteins were found to have comparable numbers of $\alpha$-helices and $\beta$-sheets, ranging from 16 to 21 and 14 to 17 . The 3D structure of CIPKs have not been fully explored in other plants, however in Prunus mume 3D structures of PmCIPK proteins were predicted which shared the highest identity with the hypothetical protein c6c9dB [50], similar to CaCIPK proteins.

\subsection{Interaction patterns between CBL and CIPK proteins in chickpea}

CIPKs are generally activated by interaction with CBLs, and then perform different functions. Thus, it is crucial to determine interactions and functional complexes of CBLs and CIPKs in chickpea. Therefore, in silico analysis was performed to analyse the CBL and CIPK interactions in chickpea. A combined score of co-expression, experimentally determined interaction and automated text mining was used to predict the strength of interaction. A score of less than 0.7 was taken as weak, whereas a score greater than 0.7 was considered as strong interaction. CaCBL1 showed strong interaction (thicker lines) with CaCIPK2, -3, -4, -6, -8, -9, -12, -14, -16, -17, -19, -22 and exhibited weak (thinner lines) interactions with CaCIPK18 and CaCIPK20 (Figure 7).

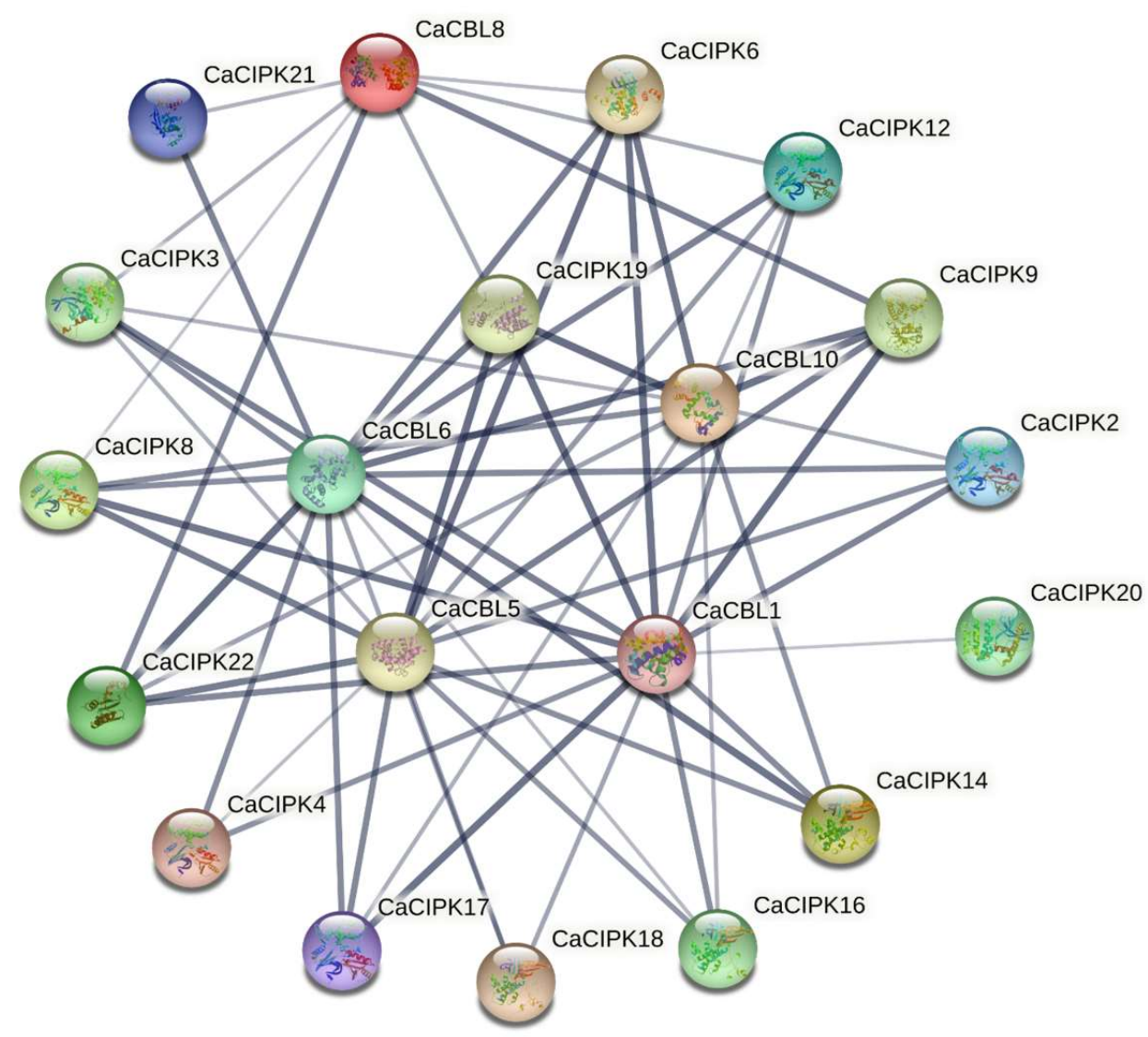

Figure 7. Interaction networks of CaCIPKs based on A.thaliana orthologues in the STRING database. The thicker and thinner lines represent strong and weak interaction respectively.

CaCBL5 showed strong interactions with nine CaCIPKs, including CaCIPK2, -6, -8, 9, -14, -16, -17, -19, -22 and weak interactions with four CaCIPKs (CaCIPK3, -4, -12 and - 
18). CaCBL6 was found to interact with 14 CaCIPKs, including CaCIPK2, $-3,-4,-6,-8,-9$, $-12,-14,-16,-17,-18,-19,-21,-22$ CaCBL8 interacted with eight CaCIPKs (CaCIPK3, -6, -8, $-9,-12,-19,21,-22)$ among which it showed strong interactions with only two CaCIPKs (CaCIPK9 and CaCIPK22). Lastly, CaCBL10 interacted with $11 \mathrm{CaCIPKs}$ among which it showed strong interactions with CaCIPK6, -8, -9, -14, -19 and weak interactions with CaCIPK2, -3, -12, -16, -17, -22 (Table S5). Interestingly, CaCIPK9 was found to interact strongly with all the CBLs. This interaction pattern suggests that each CaCIPK may interact with multiple CaCBLs and vice versa. Such interactions may determine functional specificity or overlap of chickpea CBL-CIPK complexes and subcellular localization of CaCIPKs in condition specific manner. Similar interaction patterns of CBL-CIPK complexes have been found in different plant species, including Arabidopsis [12] and rice [65]. This suggests that CBLs and CIPKs make diverse complexes to display functional specificity and synergism across plant species.

\subsection{Expression profile of CIPK genes in different developmental stages}

The expression analysis of CaCIPKs was carried out in 27 tissues of chickpea belonging to different stages i.e. germination stage (radicle, plumule, embryo), seedling stage (epicotyl, primary root), vegetative stage (root, petiole, stem, leaf), reproductive stage (nodules, flowers, buds, pods, immature seeds), and senescence stage (yellow leaf, immature seeds, mature seeds, seed coat, and nodules) (Figure 8).

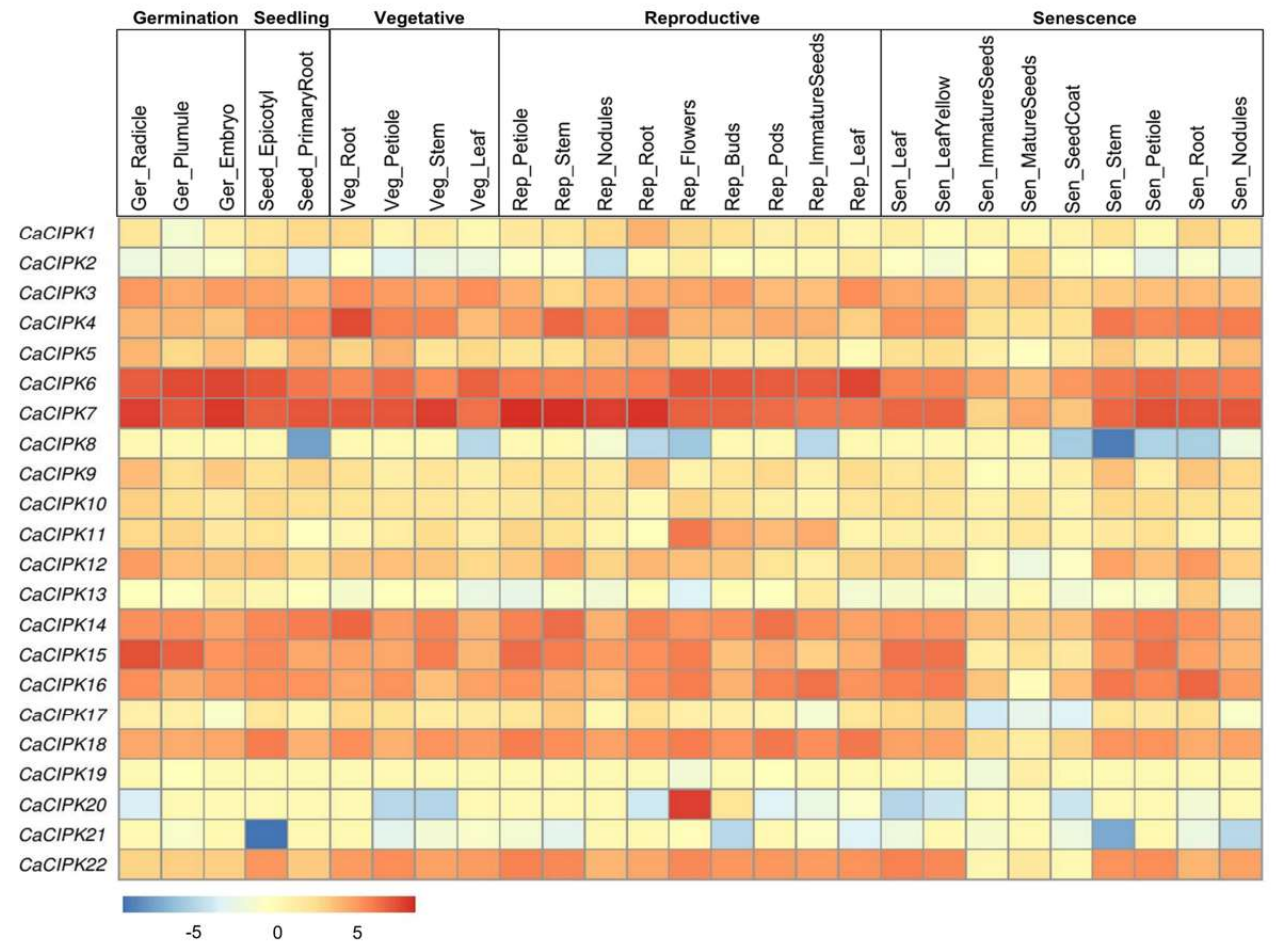

Figure 8. Expression profiles of different CIPK genes in different developmental stages of chickpea. The heatmap represents the expression pattern of CaCIPK genes in different tissues of developmental stages such as germination, seedling, vegetative, reproduction and senescence. The genes are named on the left and different tissues/developmental stages are labelled at the top. Scale bar represents the normalised log_2 FPKM values.

The results indicate that most $\mathrm{CaCIPK}$ genes show significant expression in multiple tissues and developmental stages. CaCIPK3, $-4,-6,-7,-14,-15,-16,-18$ and -22 were found 
to have ubiquitously high expression in all the tissues. Whereas CaCIPK2, $-8,-13,-17$ and -21 showed low expression in almost all the tissues. This expression pattern indicates that CIPKs might be involved in regulation of a wide array of processes during different stages of plant development in chickpea. (Table S6). Similar expression pattern has been observed for CIPKs in plants such as Arabidopsis, rice and wheat [47,65] AtCIPK19 has been found to express highly in pollen grains and pollen tubes and analysis of atcipk19 mutant and overexpression in plants revealed that AtCIPK19 is required for pollen tube growth and polarity [24]. AtCIPK6 and its chickpea ortholog have been shown to regulate root development via controlling auxin transport in Arabidopsis [26]. Tomato SICIPK2 is specifically expressed in floral organs and through interaction with different SICBLs and transcription factors regulates stamen development and stress tolerance [85]. Also, Manihot esculenta CIPKs; MeCIPK16 and MeCIPK20 were predominantly expressed in flowers [86]. These findings suggest that CIPKs are key regulators of plant development.

\subsection{Expression profile of CIPK genes in abiotic stress}

To investigate the possible involvement of chickpea CIPKs in abiotic stress signaling, their expression pattern was analysed in root and shoot under three major abiotic stresses, desiccation, salinity and cold. Several CIPK genes were found to differentially express both in root and shoot (Figure 9).

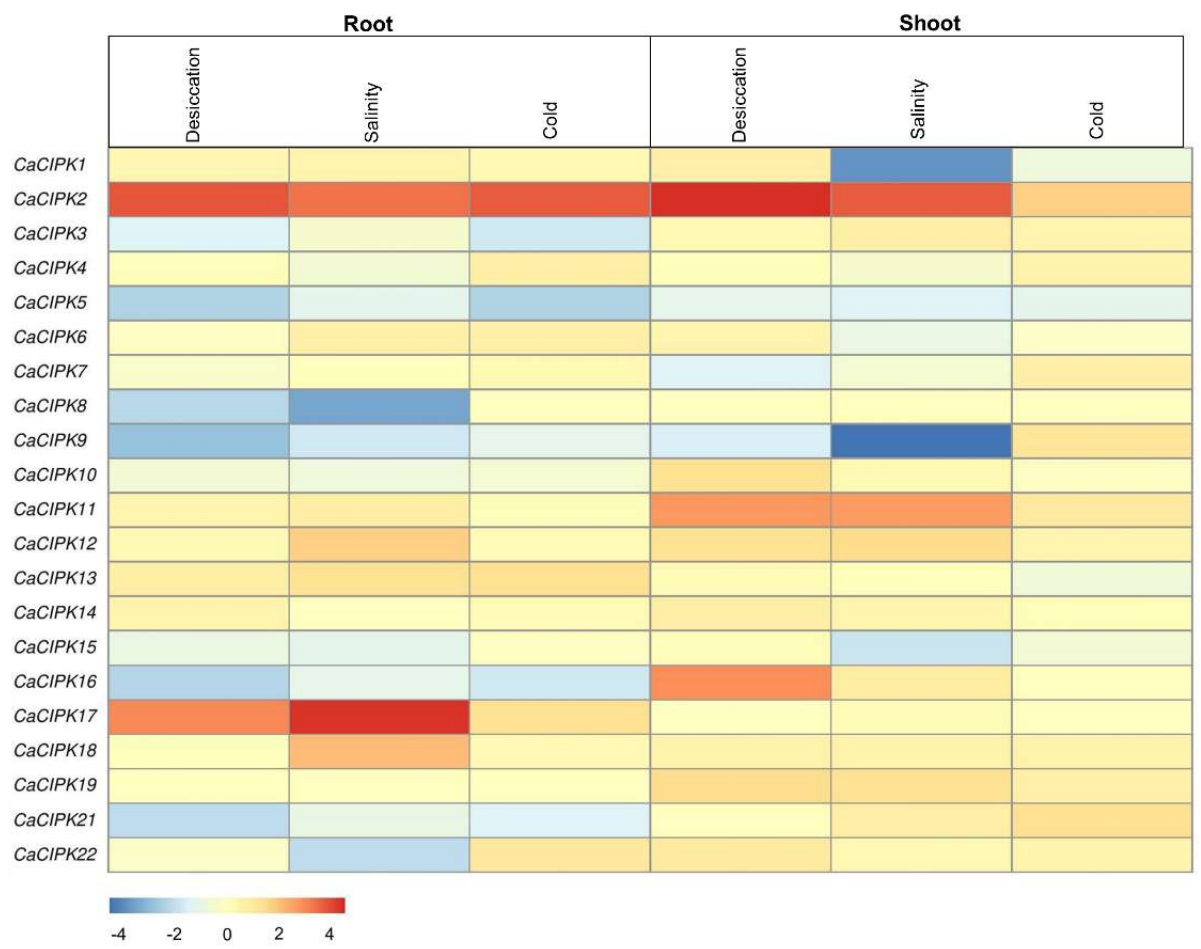

Figure 9. Expression profiles of CIPK genes of chickpea in three abiotic stress - cold, desiccation and salinity in root and shoot. The genes are named on the left and condition/tissue are labelled at the top. The scale bar represents the log_2 Fold Change based on FPKM values.

Seven genes, CaCIPK2, $-4,-6-11,-12,-13$, and -18 were found to be commonly upregulated in all three abiotic stresses, whereas five genes CaCIPK3, -5, -9, -16 and -21 were commonly downregulated in root (Table S7). Notably, CaCIPK8 was upregulated in cold 
stress but commonly downregulated in desiccation and salinity stresses. Similarly, eight genes CaCIPK2, -10, -11, -12, -14, -16, -18 and -19 were commonly upregulated in multiple stresses in shoot. Only a single gene CaCIPK5 was commonly downregulated in all stresses in shoot. CaCIPK7 and -9 were commonly downregulated in desiccation and salinity but upregulated in cold stress. Similarly, CaCIPK1 and - 15 were commonly downregulated in salinity and cold stresses but upregulated in desiccation. CaCIPK2 was ubiquitously expressed in all the stresses, both in root and shoot. These results suggest that several CaCIPK genes may have an important role in abiotic stress signaling in chickpea. Previously, CIPKs from different plant species have been implicated in abiotic stress responses. For example, salt overly sensitive (SOS) pathway is well characterized in Arabidopsis, where membrane localized AtCBL4 (SOS2) interact and activate AtCIPK24 (SOS2), which in turn regulated membrane localized $\mathrm{Na}+\mathrm{H}+$ antiporter (SOS1) to confer salt tolerance [87]. In addition, AtCIPK8, the homolog of AtCIPK24 interacts with AtCBL10 and activates SOS1 leading to salt tolerance [88]. AtCIPK21 is readily induced under salt and osmotic stress. AtCBL2 and AtCBL3 interact with AtCIPK21 and target it to the tonoplast. Analysis of AtCIPK21 overexpression and null mutant plants showed that it is a positive regulator of salt and osmotic stress tolerance in Arabidopsis [89]. Similarly, OsCIPK23 and ZmCIPK8 have been known to positively regulate drought tolerance in rice and maize respectively [90, 91].

\subsection{Expression profile in different stages of seed development}

Optimum development of seeds leads to their production with sufficient quantity as well as quality, thereby it determines the yield. To understand the role of CIPKs in chickpea seed development, expression profile was generated with mature leaf as control and seven different seed stages, representing early-embryogenesis (S1), mid-embryogenesis (S2), late-embryogenesis (S3), mid-maturation (S4-S5), and late-maturation (S6-S7), in two desi cultivars: JGK3 (large seeded) and Himchana1(small seeded) (Figure 10).

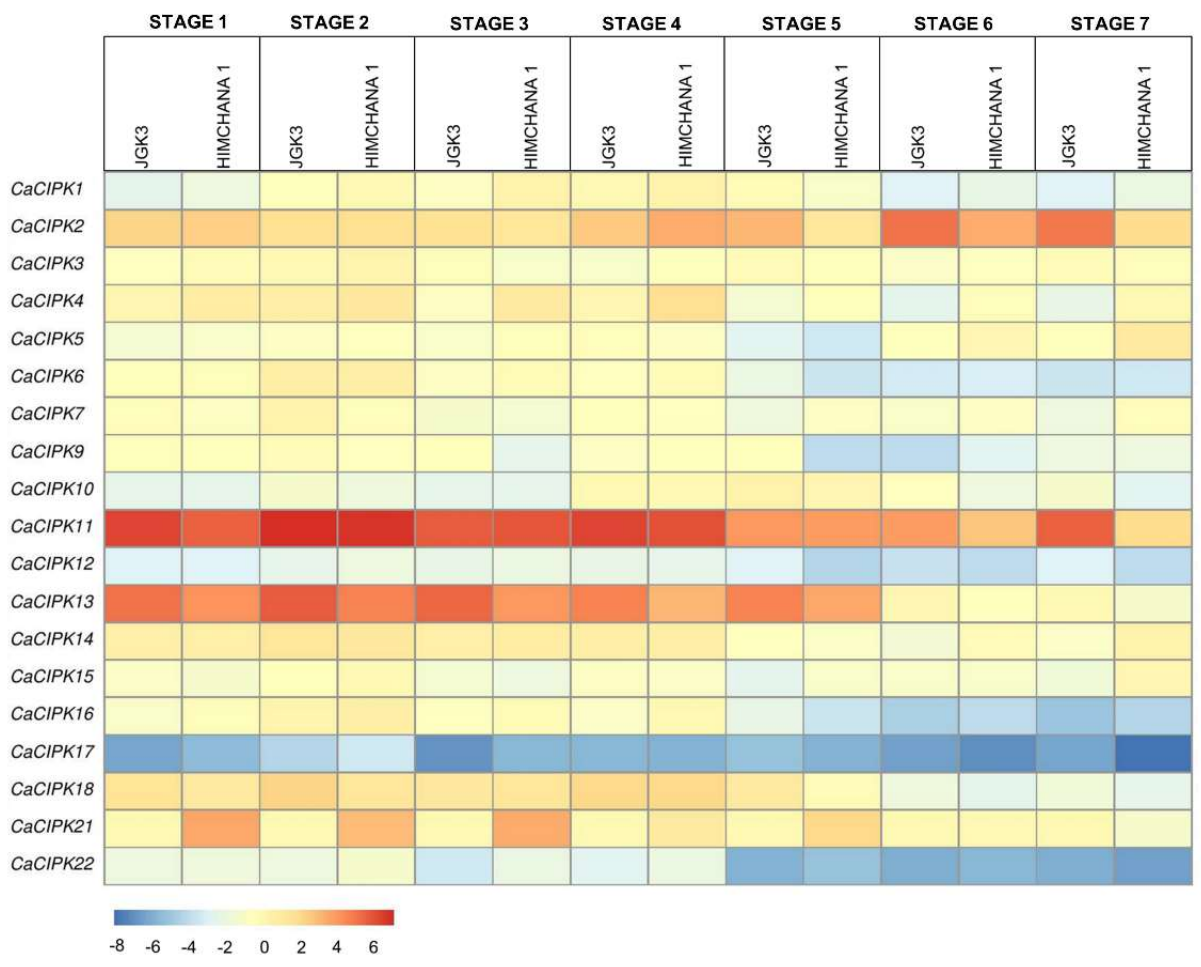


Figure 10. Expression profiles of CIPK genes in different stages of seed in chickpea in two cultivars: JGK3 and Himchana1. The genes are named on the left and stages/cultivars are labelled at the top. The scale bar represents the log_2 Fold Change based on FPKM values.

Few CaCIPK genes, including CaCIPK2, -11, -13 were ubiquitously expressed during all the seed stages in both the chickpea varieties however, level of expression varied (Table S8). CaCIPK2 expressed highly during S5-S7 in JGK3 whereas, during S4 in Himchana1. CaCIPK11 showed high expression during S1-S5 in both the varieties and during S7 in Himchana1. Similarly, CaCIPK13 showed significant expression during S1-S5 however, the level of expression was higher in JGK3 than Himchana1. In contrast, CaCIPK18 and 21 showed significant expression during S1-S5, but expression was higher in Himchana1. Remarkably, CaCIPK6 and -16 were upregulated during S1-S4 but downregulated during S5-S7 in both the varieties. CaCIPK10 was upregulated during S4-S5 in both the varieties but upregulated during S6 only in JGK3. Two CaCIPK members, CaCIPK12 and -17 were significantly downregulated during all seed stages in both JGK3 and Himchana1. These findings suggest the crucial role of CIPKs in chickpea seed development. Some CIPKs might be involved in regulating all the seed development stages in both the varieties, whereas some members might regulate specific seed stages in both varieties or any specific variety. Thus, CIPKs could play an important role in determining the chickpea yield.

Very few studies have analysed the role of CIPKs in seed development till date. The role of CIPKs in seed development has been proposed in plant species like rice and Phaseolus vulgaris. Along with CBLs, several rice CIPK genes were differentially expressed during five stages of seed development [65]. In Phaseolus vulgaris, PvCIPK1, -2, -3 and -5 were expressed only in small and mid-size developing seeds but show no expression in large developing seeds [92].

\section{Conclusions}

CIPKs have been studied at the genome-wide scale in diverse plant species, however, in-depth study of the CIPK gene family was missing in important legume crop chickpea. Therefore, in this study, genome-wide identification and characterization of the CIPK gene family was carried out in chickpea which unearthed a total of 22 CIPK genes. Gene and protein structure analysis indicated structural conservation among chickpea CIPKs and homology with other plant species. Phylogenetic analysis suggests that chickpea CIPKs have evolved from common dicot ancestors and gene duplication is the major driving force behind their evolution and expansion. Subcellular analysis showed that the CIPK proteins are majorly located in the nucleus and cytoplasm. In-silico interaction analysis revealed various specific and overlapping functional complex formations between CBLs and CIPKs which could be tested functionally in future. Expression analysis during various developmental stages indicated that CIPKs are expressed in a wide range of tissue/organs and could play an important role in their development. Promoter and expression analysis of the CIPK gene family strongly suggest their role in abiotic stress signaling and seed development in chickpea. Thus, this study provides a useful platform for detailed functional characterization of the CIPK family in chickpea and other legume crops.

Supplementary Materials: Table S1: Sequence logo of different motifs identified through MEME; Table S2: List of segmentally duplicated gene pairs; Table S3: List of cis-regulatory elements identified in the promoter regions of CaCIPK genes; Table S4: The confidence, coverage and sequence identities of the homologous relationship of the CaCIPKs; Table S5: Type and strength of interactions between CBL and CIPK proteins in chickpea; Table S6: Log2FPKM values of different tissues belonging to different developmental stages; Table S7: Log2 Fold Change values of CaCIPKs in response to abiotic stress; Table S8: Log2 Fold Change values of CaCIPKs in various stages of seed development; Figure S1: Percentage of identity and similarity between CaCIPKs; Figure S2: 
Alignment of 22 CaCIPKs for domain identification; Figure S3: Identification of motifs through MEME; Figure S4: Duplication of chickpea CIPK genes performed by MCScanX is shown via Circos plot.

Author Contributions: Conceptualization, A.S. and S.K.; data curation, N.P.; writing-original draft preparation, N.P.; writing - review and editing, A.S. and S.K.; supervision, S.K.; project administration, S.K.; funding acquisition, S.K. All authors have read and agreed to the published version of the manuscript.

Funding: This research received no external funding.

Institutional Review Board Statement: Not applicable.

Informed Consent Statement: Not applicable.

Acknowledgments: The authors are thankful to the Department of Biotechnology (DBT)-eLibrary Consortium, India, for providing access to e-resources. The authors acknowledge the Distributed Information Sub-Centers of DBT at the National Institute of Plant Genome Research (NIPGR).

Conflicts of Interest: The authors declare no conflict of interest.

\section{References}

1. 1. Luan S. The CBL-CIPK network in plant calcium signaling. Trends Plant Sci. 2009;14.

2. 2. DeFalco TA, Bender KW, Snedden WA. Breaking the code: Ca2+ sensors in plant signalling. Biochem J. $2010 ; 425$.

3. 3. Dodd AN, Kudla J, Sanders D. The Language of Calcium Signaling. Annu Rev Plant Biol. 2010;61.

4. 4. Sanders D, Pelloux J, Brownlee C, Harper JF. Calcium at the Crossroads of Signaling. Plant Cell. $2002 ; 14$ suppl 1.

5. 5. Kudla J, Becker D, Grill E, Hedrich R, Hippler M, Kummer U, et al. Advances and current challenges in calcium signaling. New Phytol. 2018;218.

6. 6. Luan S, Kudla J, Rodriguez-Concepcion M, Yalovsky S, Gruissem W. Calmodulins and Calcineurin B-like Proteins. Plant Cell. 2002;14 suppl 1.

7. 7. Tang R-J, Wang C, Li K, Luan S. The CBL-CIPK Calcium Signaling Network: Unified Paradigm from 20 Years of Discoveries. Trends Plant Sci. 2020;25.

8. 8. Wang JP, Munyampundu JP, Xu YP, Cai XZ. Phylogeny of plant calcium and calmodulin-dependent protein kinases (CcaMKs) and functional analyses of tomato CCaMK in disease resistance. Front Plant Sci. 2015;6 DEC. doi:10.3389/fpls.2015.01075.

9. 9. Singh A, Sagar S, Biswas DK. Calcium Dependent Protein Kinase, a Versatile Player in Plant Stress Management and Development. CRC Crit Rev Plant Sci. 2017;36.

10. 10. Shi J, Kim KN, Ritz O, Albrecht V, Gupta R, Harter K, et al. Novel protein kinases associated with calcineurin B-like calcium sensors in Arabidopsis. Plant Cell. 1999;11:2393-405. doi:10.1105/tpc.11.12.2393.

11. 11. Kolukisaoglu Ü, Weinl S, Blazevic D, Batistic O, Kudla J. Calcium Sensors and Their Interacting Protein Kinases: Genomics of the Arabidopsis and Rice CBL-CIPK Signaling Networks. Plant Physiol. 2004;134:43-58. doi:10.1104/pp.103.033068.

12. 12. Kim K-N, Cheong YH, Gupta R, Luan S. Interaction Specificity of Arabidopsis Calcineurin B-Like Calcium Sensors and Their Target Kinases. Plant Physiol. 2000;124.

13. 13. Albrecht V, Ritz O, Linder S, Harter K, Kudla J. The NAF domain defines a novel protein-protein interaction module conserved in Ca2+-regulated kinases. EMBO J. 2001;20:1051-63. doi:10.1093/emboj/20.5.1051.

14. 14. Guo Y, Halfter U, Ishitani M, Zhu J-K. Molecular Characterization of Functional Domains in the Protein Kinase SOS2 That Is Required for Plant Salt Tolerance. Plant Cell. 2001;13:1383-400. doi:10.1105/tpc.010021.

15. 15. Kurusu T, Hamada J, Hamada H, Hanamata S, Kuchitsu K. Roles of calcineurin B-like protein-interacting protein kinases in innate immunity in rice. Plant Signal Behav. 2010;5:1045-7. doi:10.4161/psb.5.8.12407.

16. 16. Yan $Y, H e X, H u$ W, Liu G, Wang P, He C, et al. Functional analysis of MeCIPK23 and MeCBL1/9 in cassava defense response against Xanthomonas axonopodis pv. manihotis. Plant Cell Rep. 2018;37:887-900. doi:10.1007/s00299-018-2276-7.

17. 17. Sardar A, Nandi AK, Chattopadhyay D. CBL-interacting protein kinase 6 negatively regulates immune response to Pseudomonas syringae in Arabidopsis. J Exp Bot. 2017;68:3573-84. doi:10.1093/jxb/erx170.

18. 18. Qiu QS, Guo Y, Dietrich MA, Schumaker KS, Zhu JK. Regulation of SOS1, a plasma membrane Na+/H+ exchanger in Arabidopsis thaliana, by SOS2 and SOS3. Proc Natl Acad Sci U S A. 2002;99:8436-41. doi:10.1073/pnas.122224699.

19. 19. Pandey GK, Kanwar P, Singh A, Steinhorst L, Pandey A, Yadav AK, et al. Calcineurin B-like protein-interacting protein kinase CIPK21 regulates osmotic and salt stress responses in Arabidopsis. Plant Physiol. 2015;169:780-92. doi:10.1104/pp.15.00623.

20. 20. Pandey GK, Cheong YH, Kim BG, Grant JJ, Li L, Luan S. CIPK9: A calcium sensor-interacting protein kinase required for low-potassium tolerance in Arabidopsis. Cell Res. 2007;17:411-21. doi:10.1038/cr.2007.39. 
21. 21. Singh A, Yadav AK, Kaur K, Sanyal SK, Jha SK, Fernandes JL, et al. A protein phosphatase 2C, AP2C1, interacts with and negatively regulates the function of CIPK9 under potassium-deficient conditions in Arabidopsis. J Exp Bot. 2018;69:4003-15. doi:10.1093/jxb/ery182.

22. 22. Tang R-J, Zhao F-G, Garcia VJ, Kleist TJ, Yang L, Zhang H-X, et al. Tonoplast CBL-CIPK calcium signaling network regulates magnesium homeostasis in Arabidopsis. Proc Natl Acad Sci. 2015;112.

23. 23. Mogami J, Fujita Y, Yoshida T, Tsukiori Y, Nakagami H, Nomura Y, et al. Two Distinct Families of Protein Kinases Are Required for Plant Growth under High External Mg 2+ Concentrations in Arabidopsis. Plant Physiol. $2015 ; 167$.

24. 24. Zhou L, Lan W, Chen B, Fang W, Luan S. A Calcium Sensor-Regulated Protein Kinase, CALCINEURIN B-LIKE PROTEININTERACTING PROTEIN KINASE19, Is Required for Pollen Tube Growth and Polarity. Plant Physiol. 2015;167.

25. 25. Comas LH, Becker SR, Cruz VM V., Byrne PF, Dierig DA. Root traits contributing to plant productivity under drought. Frontiers in Plant Science. 2013;4 NOV. doi:10.3389/fpls.2013.00442.

26. 26. Tripathi V, Parasuraman B, Laxmi A, Chattopadhyay D. CIPK6, a CBL-interacting protein kinase is required for development and salt tolerance in plants. Plant J. 2009;58:778-90. doi:10.1111/j.1365-313X.2009.03812.x.

27. 27. Kumar Meena M, Kumar Vishwakarma N, Tripathi V, Chattopadhyay D. CBL-interacting protein kinase 25 contributes to root meristem development. J Exp Bot. 2019;70:133-47. doi:10.1093/jxb/ery334.

28. 28. Meena MK, Ghawana S, Sardar A, Dwivedi V, Khandal H, Roy R, et al. Investigation of genes encoding calcineurin B-like protein family in legumes and their expression analyses in chickpea (Cicer arietinum L.). PLoS One. 2015;10.

29. 29. Li W, Godzik A. Cd-hit: a fast program for clustering and comparing large sets of protein or nucleotide sequences. Bioinformatics. 2006;22.

30. 30. Quevillon E, Silventoinen V, Pillai S, Harte N, Mulder N, Apweiler R, et al. InterProScan: protein domains identifier. Nucleic Acids Res. 2005;33 Web Server.

31. 31. Thompson JD, Higgins DG, Gibson TJ. CLUSTAL W: improving the sensitivity of progressive multiple sequence alignment through sequence weighting, position-specific gap penalties and weight matrix choice. Nucleic Acids Res. 1994;22.

32. 32. Kumar S, Stecher G, Li M, Knyaz C, Tamura K. MEGA X: Molecular Evolutionary Genetics Analysis across Computing Platforms. Mol Biol Evol. 2018;35.

33. 33. Letunic I, Bork P. Interactive Tree Of Life (iTOL): an online tool for phylogenetic tree display and annotation. Bioinformatics. 2007;23.

34. 34. Bailey TL, Williams N, Misleh C, Li WW. MEME: discovering and analyzing DNA and protein sequence motifs. Nucleic Acids Res. 2006;34 Web Server.

35. 35. Liu W, Xie Y, Ma J, Luo X, Nie P, Zuo Z, et al. IBS: an illustrator for the presentation and visualization of biological sequences: Fig. 1. Bioinformatics. 2015;31.

36. 36. Chen C, Chen H, Zhang Y, Thomas HR, Frank MH, He Y, et al. TBtools: An Integrative Toolkit Developed for Interactive Analyses of Big Biological Data. Mol Plant. 2020;13.

37. 37. Wang Y, Tang H, DeBarry JD, Tan X, Li J, Wang X, et al. MCScanX: a toolkit for detection and evolutionary analysis of gene synteny and collinearity. Nucleic Acids Res. 2012;40.

38. 38. Suyama M, Torrents D, Bork P. PAL2NAL: robust conversion of protein sequence alignments into the corresponding codon alignments. Nucleic Acids Res. 2006;34 Web Server.

39. 39. Yu C-S, Chen Y-C, Lu C-H, Hwang J-K. Prediction of protein subcellular localization. Proteins Struct Funct Bioinforma. 2006;64.

40. 40. Gasteiger E, Hoogland C, Gattiker A, Duvaud S, Wilkins MR, Appel RD, et al. Protein Identification and Analysis Tools on the ExPASy Server. In: The Proteomics Protocols Handbook. Totowa, NJ: Humana Press; 2005.

41. 41. Kelley LA, Mezulis S, Yates CM, Wass MN, Sternberg MJE. The Phyre2 web portal for protein modeling, prediction and analysis. Nat Protoc. 2015;10.

42. 42. Chen S, Zhou Y, Chen Y, Gu J. fastp: an ultra-fast all-in-one FASTQ preprocessor. Bioinformatics. 2018;34.

43. 43. Kim D, Paggi JM, Park C, Bennett C, Salzberg SL. Graph-based genome alignment and genotyping with HISAT2 and HISATgenotype. Nat Biotechnol. 2019;37.

44. 44. Pertea M, Pertea GM, Antonescu CM, Chang T-C, Mendell JT, Salzberg SL. StringTie enables improved reconstruction of a transcriptome from RNA-seq reads. Nat Biotechnol. 2015;33.

45. 45. Drerup MM, Schlücking K, Hashimoto K, Manishankar P, Steinhorst L, Kuchitsu K, et al. The calcineurin B-like calcium sensors CBL1 and CBL9 together with their interacting protein kinase CIPK26 regulate the arabidopsis NADPH oxidase RBOHF. Mol Plant. 2013;6:559-69.

46. 46. Merlin L, Prajeesh T, Binesh M, Dileep K. Characterization of Drought Responsive Genes of CIPK Families in Rice, Maize and Sorghum. J Rice Res Dev. 2020;3.

47. 47. Sun T, Wang Y, Wang M, Li T, Zhou Y, Wang X, et al. Identification and comprehensive analyses of the CBL and CIPK gene families in wheat (Triticum aestivum L.). BMC Plant Biol. 2015;15.

48. 48. Zhang Y, Zhou X, Liu S, Yu A, Yang C, Chen X, et al. Identification and Functional Analysis of Tomato CIPK Gene Family. Int J Mol Sci. 2019;21:110. doi:10.3390/ijms21010110.

49. 49. Zhang H, Yang B, Liu WZ, Li H, Wang L, Wang B, et al. Identification and characterization of CBL and CIPK gene families in canola (Brassica napus L.). BMC Plant Biol. 2014;14:1-24. doi:10.1186/1471-2229-14-8. 
50. 50. Li P, Zheng T, Li L, Zhuo X, Jiang L, Wang J, et al. Identification and comparative analysis of the CIPK gene family and characterization of the cold stress response in the woody plant Prunus mume. PeerJ. 2019;7.

51. 51. Ma X, Gai W-X, Qiao Y-M, Ali M, Wei A-M, Luo D-X, et al. Identification of CBL and CIPK gene families and functional characterization of CaCIPK1 under Phytophthora capsici in pepper (Capsicum annuum L.). BMC Genomics. $2019 ; 20$.

52. 52. Liu J, Chen N, Chen F, Cai B, Dal Santo S, Tornielli GB, et al. Genome-wide analysis and expression profile of the bZIP transcription factor gene family in grapevine (Vitis vinifera). BMC Genomics. 2014;15:1-18. doi:10.1186/1471-2164-15-281.

53. 53. Wang N, Zheng Y, Xin H, Fang L, Li S. Comprehensive analysis of NAC domain transcription factor gene family in Vitis vinifera. Plant Cell Rep. 2013;32:61-75. doi:10.1007/s00299-012-1340-y.

54. 54. Yu Y, Xia X, Yin W, Zhang H. Comparative genomic analysis of CIPK gene family in Arabidopsis and Populus. Plant Growth Regul. 2007;52:101-10.

55. 55. Xiang Y, Huang Y, Xiong L. Characterization of stress-responsive CIPK genes in rice for stress tolerance improvement. Plant Physiol. 2007;144:1416-28. doi:10.1104/pp.107.101295.

56. 56. Zhu K, Chen F, Liu J, Chen X, Hewezi T, Cheng Z-M. Evolution of an intron-poor cluster of the CIPK gene family and expression in response to drought stress in soybean. Sci Rep. 2016;6.

57. 57. Su W, Ren Y, Wang D, Huang L, Fu X, Ling H, et al. New insights into the evolution and functional divergence of the CIPK gene family in Saccharum. BMC Genomics. 2020;21:868. doi:10.1186/s12864-020-07264-9.

58. 58. Chaves-Sanjuan A, Sanchez-Barrena MJ, Gonzalez-Rubio JM, Moreno M, Ragel P, Jimenez M, et al. Structural basis of the regulatory mechanism of the plant CIPK family of protein kinases controlling ion homeostasis and abiotic stress. Proc Natl Acad Sci U S A. 2014;111:E4532-41. doi:10.1073/pnas.1407610111.

59. 59. Sanyal SK, Mahiwal S, Nambiar DM, Pandey GK. CBL-CIPK module-mediated phosphoregulation: facts and hypothesis. Biochem J. 2020;477.

60. 60. Ohta M, Guo Y, Halfter U, Zhu JK. A novel domain in the protein kinase SOS2 mediates interaction with the protein phosphatase 2C ABI2. Proc Natl Acad Sci U S A. 2003;100:11771-6. doi:10.1073/pnas.2034853100.

61. 61. Chen X, Gu Z, Xin D, Hao L, Liu C, Huang J, et al. Identification and characterization of putative CIPK genes in maize. J Genet Genomics. 2011;38.

62. 62. LI L bin, ZHANG Y rong, LIU K chang, NI Z fu, FANG Z jun, SUN Q xin, et al. Identification and Bioinformatics Analysis of SnRK2 and CIPK Family Genes in Sorghum. Agric Sci China. 2010;9:19-30.

63. 63. Niu L, Dong B, Song Z, Meng D, Fu Y. Genome-Wide Identification and Characterization of CIPK Family and Analysis Responses to Various Stresses in Apple (Malus domestica). Int J Mol Sci. 2018;19:2131. doi:10.3390/ijms19072131.

64. 64. Chen XF, Gu ZM, Liu F, Ma BJ, Zhang HS. Molecular Analysis of Rice CIPKs Involved in Both Biotic and Abiotic Stress Responses. Rice Sci. 2011;18:1-9.

65. 65. Kanwar P, Sanyal SK, Tokas I, Yadav AK, Pandey A, Kapoor S, et al. Comprehensive structural, interaction and expression analysis of CBL and CIPK complement during abiotic stresses and development in rice. Cell Calcium. 2014;56:81-95.

66. 66. Xi Y, Liu J, Dong C, Cheng Z-M (Max). The CBL and CIPK Gene Family in Grapevine (Vitis vinifera): Genome-Wide Analysis and Expression Profiles in Response to Various Abiotic Stresses. Front Plant Sci. 2017;8:978. doi:10.3389/fpls.2017.00978.

67. 67. Jeffares DC, Tomiczek B, Sojo V, dos Reis M. A beginners guide to estimating the non-synonymous to synonymous rate ratio of all protein-coding genes in a genome. Methods Mol Biol. 2015;1201:65-90. doi:10.1007/978-1-4939-1438-8_4.

68. 68. Baker SS, Wilhelm KS, Thomashow MF. The 5'-region of Arabidopsis thaliana cor15a has cis-acting elements that confer cold-, drought- and ABA-regulated gene expression. Plant Mol Biol. 1994;24:701-13. doi:10.1007/BF00029852.

69. 69. Germain H, Lachance D, Pelletier G, Fossdal CG, Solheim H, Séguin A. The expression pattern of the Picea glauca Defensin 1 promoter is maintained in Arabidopsis thaliana, indicating the conservation of signalling pathways between angiosperms and gymnosperms*. J Exp Bot. 2012;63:785-95. doi:10.1093/jxb/err303.

70. 70. Hernandez-Garcia CM, Finer JJ. Identification and validation of promoters and cis-acting regulatory elements. Plant Science. 2014;217-218:109-19.

71. 71. Mohanty B, Krishnan SPT, Swarup S, Bajic VB. Detection and preliminary analysis of motifs in promoters of anaerobically induced genes of different plant species. Ann Bot. 2005;96:669-81. doi:10.1093/aob/mci219.

72. 72. Sakai T, Takahashi Y, Nagata T. Analysis of the Promoter of the Auxin-Inducible Gene, parC, of Tobacco. 1996. https://academic.oup.com/pcp/article/37/7/906/1856327. Accessed 4 Jan 2021.

73. 73. Abe H, Yamaguchi-Shinozaki K, Urao T, Iwasaki T, Hosokawa D, Shinozaki K. Role of Arabidopsis MYC and MYB homologs in drought- and abscisic acid-regulated gene expression. Plant Cell. 1997;9:1859-68. doi:10.1105/tpc.9.10.1859.

74. 74. Park SC, Kwon H Bin, Shih MC. Cis-acting elements essential for light regulation of the nuclear gene encoding the a subunit of chloroplast glyceraldehyde 3-phosphate dehydrogenase in Arabidopsis thaliana. Plant Physiol. 1996;112:1563-71. doi:10.1104/pp.112.4.1563.

75. 75. Kaur A, Pati PK, Pati AM, Nagpal AK. In-silico analysis of cis-acting regulatory elements of pathogenesis-related proteins of Arabidopsis thaliana and Oryza sativa. PLoS One. 2017;12. doi:10.1371/journal.pone.0184523.

76. 76. Choi C, Hwang S-H, Fang IR, Kwon S Il, Park SR, Ahn I, et al. Molecular characterization of Oryza sativa WRKY6, which binds to W-box-like element 1 of the Oryza sativa pathogenesis-related ( PR ) 10a promoter and confers reduced susceptibility to pathogens. New Phytol. 2015;208:846-59. doi:10.1111/nph.13516. 
77. 77. Hattori T, Terada T, Hamasuna S. Regulation of the Osem gene by abscisic acid and the transcriptional activator VP1: analysis of cis-acting promoter elements required for regulation by abscisic acid and VP1. Plant J. 1995;7:913-25. doi:10.1046/j.1365313X.1995.07060913.x.

78. 78. Hobo T, Asada M, Kowyama Y, Hattori T. ACGT-containing abscisic acid response element (ABRE) and coupling element 3 (CE3) are functionally equivalent. Plant J. 1999;19:679-89. doi:10.1046/j.1365-313X.1999.00565.x.

79. 79. Batistič O, Waadt R, Steinhorst L, Held K, Kudla J. CBL-mediated targeting of CIPKs facilitates the decoding of calcium signals emanating from distinct cellular stores. Plant J. 2010;61:211-22. doi:10.1111/j.1365-313X.2009.04045.x.

80. 80. Weinl S, Kudla J. The CBL-CIPK Ca2+-decoding signaling network: Function and perspectives. New Phytologist. 2009;184:517-28. doi:10.1111/j.1469-8137.2009.02938.x.

81. 81. Kim BG, Waadt R, Cheong YH, Pandey GK, Dominguez-Solis JR, Schültke S, et al. The calcium sensor CBL10 mediates salt tolerance by regulating ion homeostasis in Arabidopsis. Plant J. 2007;52:473-84. doi:10.1111/j.1365-313X.2007.03249.x.

82. 82. D'Angelo C, Weinl S, Batistic O, Pandey GK, Cheong YH, Schültke S, et al. Alternative complex formation of the Ca 2+ regulated protein kinase CIPK1 controls abscisic acid-dependent and independent stress responses in Arabidopsis. Plant J. 2006;48.

83. 83. Batistič O, Sorek N, Schültke S, Yalovsky S, Kudla J. Dual Fatty Acyl Modification Determines the Localization and Plasma Membrane Targeting of CBL/CIPK Ca 2+ Signaling Complexes in Arabidopsis. Plant Cell. 2008;20.

84. 84. Xu J, Li H-D, Chen L-Q, Wang Y, Liu L-L, He L, et al. A Protein Kinase, Interacting with Two Calcineurin B-like Proteins, Regulates K+ Transporter AKT1 in Arabidopsis. Cell. 2006;125.

85. 85. Yuasa T, Ishibashi Y, Iwaya-Inoue M. A Flower Specific Calcineurin B-Like Molecule (CBL)-Interacting Protein Kinase (CIPK) Homolog in Tomato Cultivar Micro-Tom (\&amp;lt;i\&amp;gt;Solanum lycopersicum\&amp;lt;/i\&amp;gt; L.). Am J Plant Sci. 2012;03.

86. 86. Mo C, Wan S, Xia Y, Ren N, Zhou Y, Jiang X. Expression Patterns and Identified Protein-Protein Interactions Suggest That Cassava CBL-CIPK Signal Networks Function in Responses to Abiotic Stresses. Front Plant Sci. 2018;9:269. doi:10.3389/fpls.2018.00269.

87. 87. Qiu Q-S, Guo Y, Dietrich MA, Schumaker KS, Zhu J-K. Regulation of SOS1, a plasma membrane Na+/H+ exchanger in Arabidopsis thaliana, by SOS2 and SOS3. Proc Natl Acad Sci. 2002;99.

88. 88. Yin X, Xia Y, Xie Q, Cao Y, Wang Z, Hao G, et al. The protein kinase complex CBL10-CIPK8-SOS1 functions in Arabidopsis to regulate salt tolerance. J Exp Bot. 2020;71.

89. 89. Pandey GK, Kanwar P, Singh A, Steinhorst L, Pandey A, Yadav AK, et al. Calcineurin B-Like Protein-Interacting Protein Kinase CIPK21 Regulates Osmotic and Salt Stress Responses in Arabidopsis. Plant Physiol. 2015;169.

90. 90. Yang W, Kong Z, Omo-Ikerodah E, Xu W, Li Q, Xue Y. Calcineurin B-like interacting protein kinase OsCIPK23 functions in pollination and drought stress responses in rice (Oryza sativa L.). J Genet Genomics. 2008;35.

91. 91. Tai F, Yuan Z, Li S, Wang Q, Liu F, Wang W. ZmCIPK8, a CBL-interacting protein kinase, regulates maize response to drought stress. Plant Cell, Tissue Organ Cult. 2016;124.

92. 92. Hamada S, Seiki Y, Watanabe K, Ozeki T, Matsui H, Ito H. Expression and interaction of the CBLs and CIPKs from immature seeds of kidney bean (Phaseolus vulgaris L.). Phytochemistry. 2009;70:501-7. 